\title{
DEM modeling of segregation and stratification in pouring heaps of bi- dispersed mixtures of rounded particles
}

\author{
Tristan Vuilloz ${ }^{1,2, *}$, David Cantor ${ }^{1,2, * *}$, and Carlos Ovalle ${ }^{1,2, * * *}$ \\ ${ }^{1}$ Department of Civil, Geological and Mining Engineering, Polytechnique Montréal, Québec, Canada \\ ${ }^{2}$ Research Institute of Mining and Environment, RIME UQAT-Polytechnique, Montréal, Québec, Canada
}

\begin{abstract}
This study aims to assess and analyse the patterns of segregation and stratification in pouring heaps of granular mixtures composed by binary sized and uniformly shaped particles. We present 2D and 3D simulations which respectively build deposits of poured disks and spheres by means of a discrete-element approach known as contact dynamics (CD). In order to identify preferable conditions for segregation and stratification, we try several deposition scenarios varying the pouring flow rate, injection height, heap's width and mass ratio between large and small grains in our binary samples. Although some authors assert that shape dispersity might not be necessary to obtain stratification, the phenomenon seems hard to seize with mono-shaped granular media as it appears to require a close control on pouring conditions. The introduction of our DEM models and statistical analysis intend to provide examples of what could constitute efficient numerical tools to study the remaining open problems related to heap segregation patterns prediction.
\end{abstract}

\section{Introduction}

Granular flows are often found in natural environments and industry as main mechanism for transport and deposit of geomaterials. Pouring grains onto heaps produces, for instance, segregation and stratification which could affect activities such as building stockpiles of crushed mined ore and dumping in mining waste rock piles.

Segregation and stratification are responsible for a distribution of particles in distinctive assemblies driven by differences of physical properties among granular samples. Segregation has been examined highlighting the role of percolation and buoyancy as the main mechanisms of splitting by grain properties $[4,20]$. Such grain kinematics have been observed in existing granular dumps [14] and studied with experiments and mathematical modeling on repeated occasions $[16,19]$. Stratification, on the other hand, is less understood. Makse et al. experimentally triggered stratification by dropping a bi-dispersed mixture composed of small glass beads and larger angular sand grains in a narrow container [12-14]. By pouring particles onto a pile, they displayed a pattern with alternating layers of glass beads and sand parallel to the free surface of the heap. The key parameter for stratification was therefore attributed to the difference of repose angles between the two particles species, leading to a competition between size and shape induced segregation. The resulting unsteady state is responsible for the creation of repeated surface avalanches building the pile upwards, a motion known as "kink". Gray et al. [8,9] verified that these avalanches act as a kinetic sieve which enforce grain rolling over the pile. In the meantime, Baxter et al. [1] drew stratification patterns from pouring binary-sized and similar shaped mixtures onto a heap, asserting that differences between repose angles or shape profiles could not be necessary to trigger the phenomenon but were only affecting the range of fill rates inducing stratification display. More recently, Benito et al. provided evidence that stratification could be drawn from pouring binary sized mixtures only made of spherical particles, supporting the previous assertions [2,3]. Their findings were obtained following a study on the influence of experimental parameters including size ratio, mass flux and dropping height. In particular, they found out that stratification, for such a mono-shape grain case, needs larger size ratios between the two families of particles, lower feeding rates, and a smaller cell width. Such results have been lately replicated by Zhang et al. in $3 \mathrm{D}$ discrete-element simulations exposing particle stratification for analogous pouring conditions [21].

In this paper, we perform 2D and 3D DEM simulations pouring binary samples of size-dispersed disks or spheres to build granular deposits. We intend to reproduce particle segregation and stratification after the pouring of mixtures involving no shape dispersity. Our numerical experiments allow us to vary and control deposition settings in attempt to display the same splitting effects as observed in the literature. We detail the numerical procedures in Sec. 2. The results are discussed in Sec. 3, including qualitative observations and quantitative assessments. We end this study with a series of conclusions and perspectives.

\footnotetext{
*e-mail: tristan.vuilloz@polymtl.ca

**e-mail: david.cantor@polymtl.ca

***e-mail: carlos.ovalle@polytml.ca

A video is available at https://doi.org/10.48448/vkaj-x869
} 


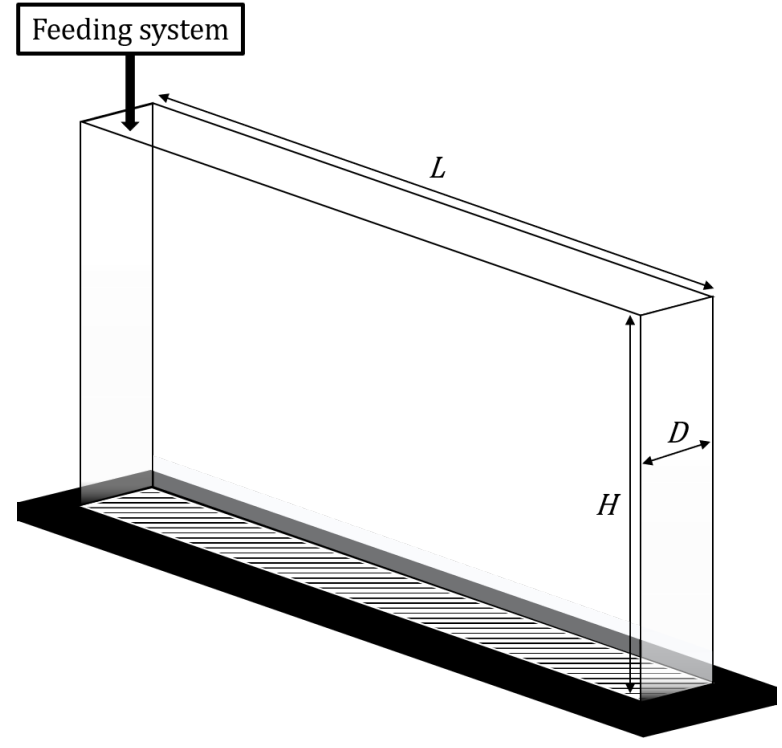

Figure 1. Testing cell representation.

\section{Numerical procedures}

We created a DEM model based on a set of experiments that intended to display stratification from granular material pouring $[2,7-9,11-14]$. Our testing device is a quasi two-dimensional cell with a fixed grain feeding system (see Fig. 1) allowing one to control the injection height and the flow rate of the deposition. Our modelling was possible with the use of the software LMGC90, developed in the University of Montpellier, which allows one to create various deformable or non deformable objects and solve the dynamics of mechanical systems by means of the contact dynamics (CD) method [18]. The CD approach [5, 10, 17] is a nonsmooth implicit numerical method in which the equations of motions are integrated for multi-contact systems with unilateral contacts and friction.

We perform the pouring of circular grains in 2D and spheres in 3D simulations using binary size distributions. The large grains have a $3 \mathrm{~mm}$ diameter while the small have a $1 \mathrm{~mm}$ diameter. Particles size ratio is thus set to $3: 1$ according to ideal stratification cases involving no shape dispersity in granular mixtures, described by Benito et al. and Zhang et al. [2, 21] or reviewed by Fan et al. [6]. Mass fill rates and free fall heights are also chosen to fit the parameters used in these previous works for discrete avalanche initiation under a slow feeding process. Each simulation is performed for a given mass index, defined as the ratio of the total mass of coarse particles over the total mass of small ones, which describes the mixture's proportion. The testing cell is reproduced with frictionless sidewalls and a rough base to prevent grains' excessive rolling. In 3D simulations, the width of the cell was varied between $D=3 \mathrm{~mm}$ or $10 \mathrm{~mm}$. We drop the grains next to a narrow face of the cell so the heap builds up being supported by the sidewalls and the rough base. We pour up to 60,000 particles at the final step of the deposition. Cell's dimensions, physical properties and pouring settings are summarized in Table 1.
Table 1. Simulations core parameters.

\begin{tabular}{||c|c||}
\hline Model's parameters & Value \\
\hline \hline Cell's length, $L(\mathrm{~mm})$ & 500 \\
Cell's height, $H(\mathrm{~mm})$ & 500 \\
Cell's width, $D(\mathrm{~mm})$ & 3 or 10 \\
\hline Particles size ratio, $s_{r}$ & $3: 1, s_{r}=1 / 3$ \\
Samples mass ratio, $m_{L S}$ & 0.5 or 1 \\
Particles density $\left(\mathrm{kg} / \mathrm{m}^{3}\right)$ & 2,700 \\
\hline Mass feeding rate, $f(\mathrm{~g} / \mathrm{s})$ & 0.1 to 0.6 \\
Injection height, $h(\mathrm{~mm})$ & 10 to 50 \\
\hline
\end{tabular}

\section{Results and discussions}

Figures 2 and 4 present screenshots from our simulations in which small grains are colored in light blue and coarse particles are colored in a dark tone.

In order to give a quantitative assessment of segregation in heaps, we introduce a mixing index $m_{I}$ which adapts to 2D and 3D geometries. The index express segregation intensity in terms of coarse particles proportion in a given pile region. We define $m_{I}$ as:

$m_{I}=\frac{n_{L}}{n_{s} \cdot s_{r}{ }^{a}+n_{L}}=\frac{m_{L S, d i v}}{m_{L S, d i v}+1} ;\left\{\begin{array}{l}a=2 \text { for 2D models } \\ a=3 \text { for 3D models }\end{array}\right.$

where $n_{L}$ and $n_{s}$ are the number of large particles and small particles, respectively, in a heap division, $s_{r}{ }^{a}$ is the volume ratio factor between small and coarse particles, and $m_{L S, d i v}$ is the mass ratio observed in a heap division.

Figures 3 and 5 summarize $m_{I}$ computations. For each pouring scenario, we divide the resulting pile in ten vertical stripes, perpendicular to the horizontal axis, of equal surface or volume. The mixing index is then computed for each one of these divisions to measure the distribution of particles sizes along the heap's length. The index $m_{I}$ turns out to be convenient as it allows one to compare mixing quality from piles differing in dimensions. The value taken by the index in each heap division may vary between 0 and 1 , from a fully segregated region containing only small particles to one with only large grains.

\subsection{Segregation}

Heap's representations (see Figs. 2 and 4) successfully display segregated patterns both in 2D and 3D simulations. Each pouring scenario seems to distribute larger particles at the bottom of the sample due to avalanche development on the free surface of the pile. The avalanches initiate a void-filling mechanism which makes small particles sink while large ones reach the upper layer of the flow. On top of the flow, coarse particles experience a greater velocity leading them to gather at the flow front as systematically observed in Refs. [8, 16]. Large particles' motion ends when the flow comes to rest on the outer parts of the granular pile while small grains early segregate inside the pile.

Those observations are additionally verified by the mixing index (see Figs. 3 and 5) which consistently shows a segregation trend across the granular piles: small particles are more likely to be found near the deposition point 
(a)

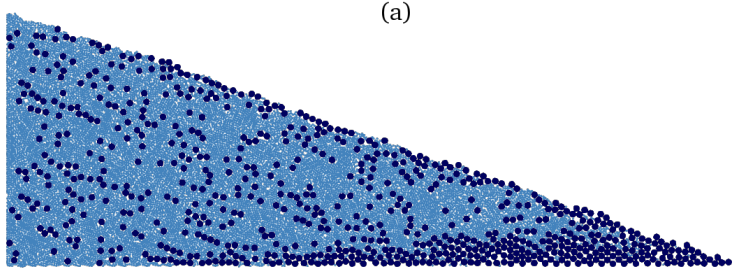

(b)

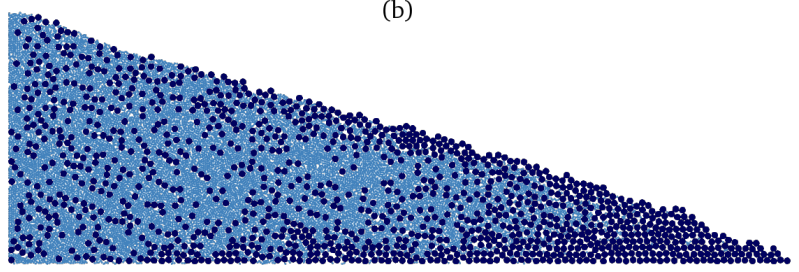

Figure 2. Heaps patterns as results of 2D simulations; (a) mass ratio $m_{L S}=0.5$, size ratio is $3: 1, f=0.1 \mathrm{~g} / \mathrm{s}$ and $h=10 \mathrm{~mm}$; (b) mass ratio $m_{L S}=1$, size ratio is $3: 1, f=0.1 \mathrm{~g} / \mathrm{s}$ and $h=10 \mathrm{~mm}$.

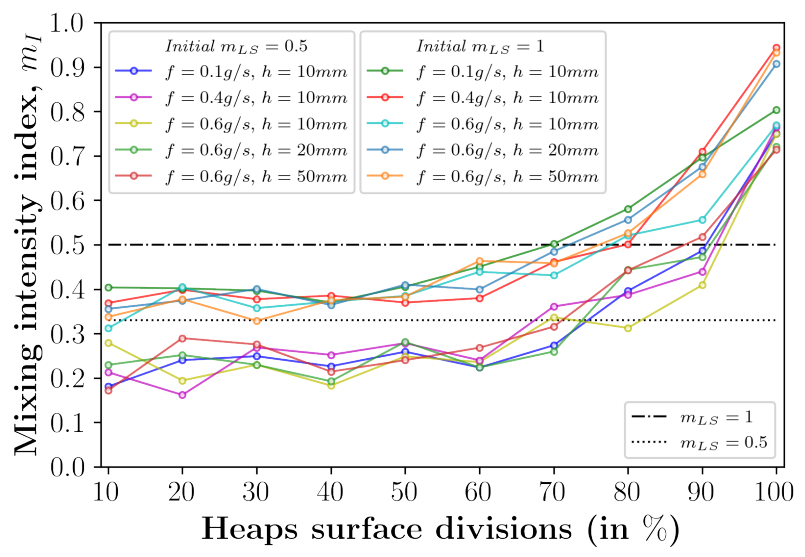

Figure 3. Mixing state computation across 2D granular deposits, heaps are divided in ten vertical stripes of same surface along its length. $m_{I}$ gradations corresponding to mixtures initial $m_{L S}$ are indicated as horizontal thresholds.

and coarse ones segregated at the tail of the heap. These observations are in agreement with the experimental studies of Benito et al. [2] and simulations of Zhang et al. [21]. Flow segregation patterns occur for injection heights varying between $10 \mathrm{~mm}$ and $50 \mathrm{~mm}$, flow rates between 0.1 and $0.6 \mathrm{~g} / \mathrm{s}$, and $m_{L S}=\{0.5,1\}$. According to both 2D and 3D simulations, the evolution of $m_{I}$ along the heap is not sensitive to changes of the flow rate neither the injection height, but is dependent on the initial $m_{L S}$.

\subsection{Stratification}

Figure 2 presents two examples showing a blurred intern distribution of grains obtained after the pouring process. The 2D model proved itself incapable of building a granular pile stable enough to constitute a static bed of particles on which the next layer forms. We observe large consecutive avalanches damaging the structures created by kinetic sieving. Therefore, an alternating layering of particles is not likely to occur. The repetition of this destructive mechanism then causes a broad mixing of the existing heap.

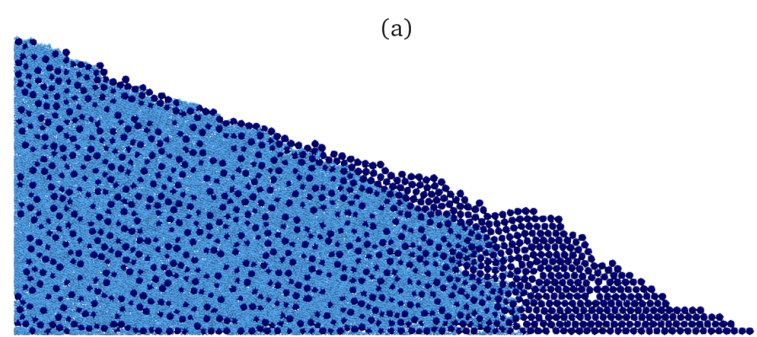

(b)

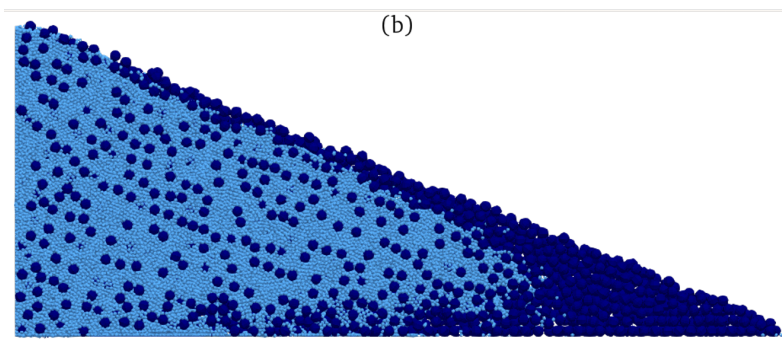

(c)

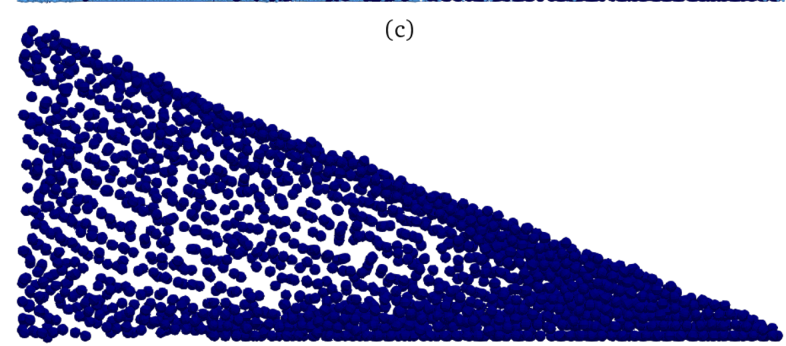

Figure 4. Heaps patterns as results of 3D simulations; (a) mass ratio $m_{L S}=1$, size ratio is $3: 1, f=0.6 \mathrm{~g} / \mathrm{s}, h=50 \mathrm{~mm}$ and $D=3 \mathrm{~mm}$, (b) mass ratio $m_{L S}=1$, size ratio is $3: 1, f=0.4 \mathrm{~g} / \mathrm{s}$, $h=10 \mathrm{~mm}$ and $D=10 \mathrm{~mm}$; then (c) presents (b) deposit with the smaller particles hidden.

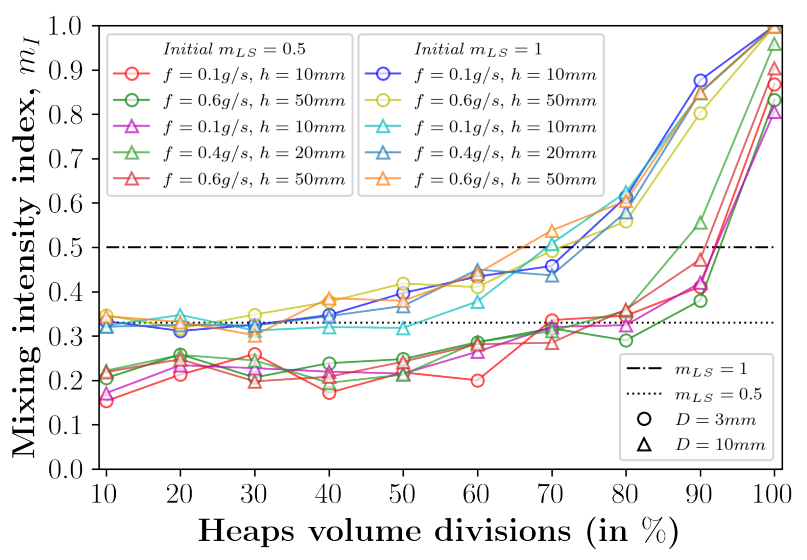

Figure 5. Mixing state computation across 3D granular deposits, heaps are divided in ten vertical stripes of same volume along its length. $m_{I}$ gradations corresponding to mixtures initial $m_{L S}$ are indicated as horizontal thresholds.

Conversely, the 3D model proves to be a better configuration to investigate stratification. The third dimension added to the testing cell helps the simulation to build a bounded heap more resilient to free surface flows development. By pouring spherical particles in a narrow container, they profit from an increased number of contacts and finite size effect which enhance their connectivity. A static 
bed of grains then progressively takes shape and surface flows are able to act as repeated kinetic sieves. The voidfilling mechanism which has been found responsible for stratification is thus triggered. Results presented in Figure 4 expose granular distributions with alternating strata of large and small particles coupled to strong concentrations of coarse segregated grains at heaps' tail. Figure 4(c) proves that such layers appear throughout the cell's width instead of a simple pattern being displayed on the walls. These patterns are similar to Zhang's et al. simulations [21] but for a broader set of deposition parameters such as those investigated by Benito et al. [2]. Yet, it remains complex to assess any stratification degree as the layers do not present similar thickness or regular separation. The fact that a close control over deposition parameters seems necessary to produce such results demonstrates non shapedispersed mixtures stratification might only be triggered in a narrow window of pouring scenarios. Since 3D models successfully reproduce stratification mechanisms and expose layering trends, we consider that clearer stratification patterns would be observed by pouring more particles to build granular piles of bigger scales. However, DEM simulations' consumption of time and resources could limit the capabilities of modeling larger collections.

\section{Conclusions}

A numerical study aiming to display segregation and stratification patterns from pouring size-dispersed granular mixtures of uniformly shaped grains onto heaps has been presented. We carried out DEM simulations in the framework of the contacts dynamics approach to create 2D and $3 \mathrm{D}$ grain piles while varying deposition parameters.

Segregation and stratification have been described qualitatively. Segregation has also been quantitatively assessed with the definition of a mixing index expressing coarse particles volume proportion in various heaps' regions. Both 2D and 3D simulations successfully displayed segregation for every deposition setting, but only 3D simulations demonstrated granular patterns which can be related to stratification. By pouring spherical particles, stratification mechanisms have been observed acting to build layered patterns. Our results support recent upstream experimental and numerical studies showing mono-shaped samples stratification in narrow testing cells. Close control over pouring parameters appeared though necessary to trigger only size-dispersed samples stratification, which should make reconsider its so-called "spontaneity". Still, mechanisms of free surface segregation have been successfully observed with the lateral growth of the heaps.

As perspective of this work, similar numerical studies involving shape-dispersed mixtures are essential to assess whether shape dispersity might remain a catalytic condition to stratification display in such pouring conditions. In order to validate such models, the investigation of heap flow kinematics is also required.

\section{Acknowledgements}

This research work benefited from the financial support of the Natural Sciences and Engineering Research Council of Canada (NSERC) [Ref. RGPIN-2019-06118], the Fonds de recherche du Québec - Nature et technologies (FRQNT) [Ref. 2020-MN-281267] and the industrial partners of the Research Institute on Mines and the Environment (RIME) UQAT-Polytechnique (irme.ca/en).

\section{References}

[1] J. Baxter, U. Tüzün, D. Heyes, I. Hayati, P. Fredlund, Nature 391, 136 (1998)

[2] J.G. Benito, I. Ippolito, A.M. Vidales, Powder. Technol. 234, 123-131 (2013)

[3] J.G. Benito, R.O. Unac, A.M. Vidales, I. Ippolito, Physica A 396, 19-28 (2014)

[4] R.I. Brown, J. I. Fuel 13, 15-19 (1939)

[5] F. Dubois, V. Acary, M. Jean, CR Mecanique 346, 247-262 (2018)

[6] Y. Fan, K.V. Jacob, B. Freireich, R.M. Lueptow, Powder. Technol. 312, 67-68 (2018)

[7] Y. Grasselli, H.J. Herrmann, Granul. Matter 1, 43-47 (1998)

[8] J.M.N.T. Gray, C. Ancey, J. Fluid Mech. 629, 387-423 (2009)

[9] J.M.N.T. Gray, K. Hutter, Continuum Mech. Therm. 9, 341-345 (1997)

[10] M. Jean, Comput. Method Appl. M. 177, 235-257 (1999)

[11] J.P. Koeppe, M. Enz, J. Kakalios, Phys. Rev. E 58, 4104-4107 (1998)

[12] H.A. Makse, S. Havlin, P.R. King, H.E. Stanley, Nature 386, 379-382 (1997)

[13] H.A. Makse, P. Cizeau, H.E. Stanley, Phys. Rev. Lett. 78, 3298-3301 (1997)

[14] H.A. Makse, Phys. Rev. E 56, $7008-7016$ (1997)

[15] R.S. Nichols, Rock segregation in waste dumps, in Proceedings of the International Symposium on Flowthrough Rock Drains, 8-11 September 1986, Cranbrook, Canada (1986)

[16] O. Pouliquen, J. Delour, S.B. Savage, Nature 386, 816-817 (1997)

[17] F. Radjai, V. Richefeu, Mech. Mater. 41, 715-728 (2009)

[18] M. Renouf, P. Alart, Comput. Method. Appl. M., 194, 2019-2041 (2005)

[19] S.B. Savage, C.K.K. Lun, J. Fluid Mech. 189, 311335 (1988)

[20] J.C. Williams, Powder. Technol. 15, 245-251 (1976)

[21] D. Zhang, Z. Zhou, D. Pinson, DEM simulation of particle stratification and segregation in stockpile formation, in Proceedings of the conference Powders and Grains, 3-7 July 2017, Montpellier, France (2017) 